\title{
Range and thermal-behavior studies of Au and Bi implanted into photoresist films
}

\author{
M. Behar, P. L. Grande, L. Amaral, J. R. Kaschny, and F. C. Zawislak \\ Instituto de Fisica, Universidade Federal do Rio Grande do Sul, 91500 Porto Alegre, Rio Grande do Sul, Brazil \\ R. B. Guimarães \\ Instituto de Fisica, Universidade Federal Fluminense, 24001 Niteroi, Rio de Janeiro, Brazil \\ J. P. Biersack and D. Fink \\ Hahn-Meitner-Institut, D-1000 Berlin, West Germany
}

(Received 17 October 1989)

\begin{abstract}
The Rutherford backscattering technique has been used to determine range parameters of $\mathrm{Au}$ and $\mathrm{Bi}$ ions implanted into AZ1350 photoresist films at energies from 20 to $300 \mathrm{keV}$. The experimental results are 20 to $25 \%$ higher than the theoretical predictions by Ziegler, Biersack, and Littmark. Good agreement is achieved only when inelastic effects are included in the nuclear stopping-power regime. In addition, we find that shallow implantation of $\mathrm{Bi}$ ions increases the temperature at which the photoresist starts to decompose. This feature is not observed when Au is implanted under the same conditions. Finally, we have studied the thermal behavior of implanted Bi and Au ions. While Bi diffuses regularly, Au does not follow an Arrhenius kind of behavior. In addition, it is shown that the implantation process modifies, via the nonannealed damage, the characteristics of the Bi diffusion behavior.
\end{abstract}

\section{INTRODUCTION}

In recent years there has been a growing interest in ion implantation of polymers, essentially because of the actual and potential applications in advanced microelectronic technology. Projected ranges $\left(R_{p}\right)$ and range stragglings $\left(\Delta R_{p}\right)$ must be known in order to determine precisely the thickness of the photoresist mask. Moreover, the knowledge of the experimental range parameters that characterize the implanted profiles are important in order to test the current range-energy theoretical predictions. Recently Ziegler, Biersack, and Littmark (ZBL) have proposed a universal potential ${ }^{1,2}$ and an improved semiempirical electronic stopping power based on the ideas of Brandt and Kitagawa. ${ }^{3}$ They are used as input in a Monte Carlo TRIM program ${ }^{4}$ in order to predict range profile parameters.

To check the ZBL predictions for complex targets like polymers, we have recently performed a systematic range study of lithium ${ }^{5}$ and boron ${ }^{6}$ implanted into the AZ111 photoresist. Our results have shown good agreement (better than $7 \%$ ) between the experimental data and the theoretical predictions. An extension of this work is to further test the ZBL calculations for heavy ions implanted into polymers, systems where there is a general lack of experimental data.

A second aspect of ion-polymer interaction that we investigate in this paper is related to the effect of ion implantation on the thermal stability of the polymers. It is known that most of the photoresists remain stable up to $200^{\circ} \mathrm{C}$. At higher temperature they start to decompose, losing molecular components like $\mathrm{H}_{2}, \mathrm{CO}_{2}, \mathrm{COH}$, etc. In an earlier work Okayama et al. ${ }^{7}$ have shown that high- energy heavily ion bombarded polymers become more resistant to heat treatment. More recently Guimarães et al. ${ }^{8}$ have shown that a shallow $\mathrm{Bi}$ implantation $\left(E=50 \mathrm{keV} ; \phi=10^{14} \mathrm{Bi} / \mathrm{cm}^{2}\right.$ ) resulted in the increase of the temperature at which the polymer starts to decompose. It was suggested, very tentatively, that the radiation damage produced by the implantation process was responsible for this behavior. However, chemical effects due to bonds between $\mathrm{Bi}$ and the atoms and molecules of the photoresist cannot be disregarded and their effects should be further explored.

A third interesting aspect investigated in this work is connected with the thermal behavior of implanted ions into polymers. To our knowledge, no related systematic studies of the diffusion of implanted species have yet been performed. Therefore questions like regularity of the diffusional process and influence of the radiation damage are open questions that should be investigated.

The thermal stability and diffusion processes are studied for $\mathrm{Bi}$ and $\mathrm{Au}$ implanted into $\mathrm{AZ} 1350$ photoresist films. Both ions have a similar mass (and therefore introduce similar radiation damage) but they possess different chemical properties. Then it should be possible to observe the influence of chemical effects on the thermal behavior of the ion-implanted samples. Finally concerning range studies, we have implanted both ions in an 20-300 $\mathrm{keV}$ energy range and compared the experimental results with the ZBL predictions. ${ }^{2}$

The Rutherford backscattering technique (RBS) was used to determine the implanted depth profiles, to observe stoichiometric changes and the diffusional behavior of the implanted species as a consequence of the thermal treatments of the photoresist. 


\section{EXPERIMENTAL PROCEDURE AND DATA ANALYSIS}

Silicon wafers were cleaned following a standard procedure. First they were submitted to a $\mathrm{SO}_{4} \mathrm{H}_{2}$ solution bath, rinsed with $\mathrm{HF}$ and submitted to a dry cleaning with an $\mathrm{N}_{2}$ jet. Then the wafers were backed at $300^{\circ} \mathrm{C}$ for $30 \mathrm{~min}$ in order to eliminate the residual water. After this, a $1.5-\mu \mathrm{m}$-thick film was spin deposited onto the wafers and finally the samples were soft baked for $25 \mathrm{~min}$ at $90^{\circ} \mathrm{C}$. Small pieces of wafers $\left(\cong 2 \mathrm{~cm}^{2}\right)$ were subsequently implanted with $\mathrm{Au}$ and $\mathrm{Bi}$ under the following conditions: (a) for range measurements with fluences of $5 \times 10^{14}$ at. $/ \mathrm{cm}^{2}$ at $20 \mathrm{keV}$ up to $2 \times 10^{15}$ at. $/ \mathrm{cm}^{2}$ at 300 $\mathrm{keV}$; (b) at a fixed energy of $50 \mathrm{keV}$ the implantation fluence was changed from $10^{14}$ to $10^{15}$ at. $/ \mathrm{cm}^{2}$ in order to investigate the effect of fluence on the range parameters; (c) for the thermal behavior studies we have implanted $\mathrm{Bi}$ and $\mathrm{Au}$ at $50 \mathrm{keV}$ at two different fluences $\phi=10^{14}$ and $3 \times 10^{14} \mathrm{at} . / \mathrm{cm}^{2}$. In all the cases the implantations were done at room temperature using the $400 \mathrm{kV}$ ion implanter at the Institute of Physics, Porto Alegre. The beam current densities were $50 \leq \mathrm{nA} / \mathrm{cm}^{2}$ in order to avoid excessive heating of the implanted samples.

Depth profiles were obtained via RBS analysis using $760 \mathrm{keV} \alpha$ particles from the same implanter. The samples were measured at two geometries: one with the beam impinging perpendicular to the sample, the second under $60^{\circ}$ with the sample's normal. The backscattered $\alpha$ particles were detected by a $\mathrm{Si}(\mathrm{Li})$ surface-barrier detector placed at $160^{\circ}$, with respect to the beam direction. The overall resolution of the system was better than 14 $\mathrm{keV}$. The beam spot on the sample was changed whenever the $\alpha$ dose reached $2 \times 10^{13}$ at. $/ \mathrm{cm}^{2}$. This procedure was followed to avoid compactation effects and formation of carbon rich regions as a consequence of large $\alpha$ irradiation fluence.

Data analysis was performed calculating directly from the measured spectra the four moments $\left[R_{p}, \Delta R_{p}, \gamma\right.$ (skewness), and $\beta$ (kurtosis)] of the ion distributions. In all the cases the implanted profiles have shown to be Gaussian with $\gamma \cong 0$ and $\beta \cong 3$. The energy to range transformation was done using the $\alpha$ stopping powers, as reported in Ref. 2. Range stragglings have been obtained after deconvolution of the measured profiles assuming that the $\alpha$ straggling and system resolution are both Gaussian. ${ }^{9}$ The main error in the evaluation of the implanted profiles is basically due to the reported uncertainty in the stopping powers, which are estimated to be around 5\% (see Ref. 10). Other sources of errors, like the instability of the electronic system, are much less important. The uncertainty introduced by the application of the Bragg's rule for the stopping power of the light and heavy ions is an open question and will be discussed in Sec. IV A.

The thermal stability of the implanted and nonimplanted samples have been investigated performing isothermal annealings of the samples at 100,200,300,350, and $400^{\circ} \mathrm{C}$ for $20 \mathrm{~min}$ each in a vacuum better than $10^{-6}$ Torr.
TABLE I. Fluence dependence of the range parameters for $50-\mathrm{keV}{ }^{209} \mathrm{Bi}$ implanted into an $1-\mu \mathrm{m}$ film of $\mathrm{AZ1350}$. The estimated oxygen loss as a consequence of the implantation process is also shown (typical error $\sim 5 \%$ ).

\begin{tabular}{cccc}
\hline \hline $\begin{array}{c}\text { Fluence } \\
\left(10^{14} \mathrm{~cm}^{-2}\right)\end{array}$ & $\begin{array}{c}R_{p} \\
(\AA)\end{array}$ & $\begin{array}{c}\Delta R_{p} \\
(\AA)\end{array}$ & $\begin{array}{c}\text { Oxygen loss } \\
(\%)\end{array}$ \\
\hline 1 & 490 & 110 & $<5$ \\
5 & 480 & 130 & 25 \\
8 & 470 & 140 & 35 \\
10 & 480 & 120 & 45 \\
\hline \hline
\end{tabular}

\section{RESULTS}

\section{A. Fluence effects}

In order to observe if the range parameters are fluence dependent, we have Bi implanted four AZ1350 samples with fluences between $10^{14}$ and $10^{15}$ at. $/ \mathrm{cm}^{2}$ all at the same energy $(50 \mathrm{keV})$. The obtained profiles are all Gaussians and their range parameters are similar, within the experimental errors, as shown in Table I. At the lowest implantation fluence, no significant modification in the chemical composition of the photoresist film has been detected-see Fig. 1(a). On the contrary for $\phi=8 \times 10^{14}$
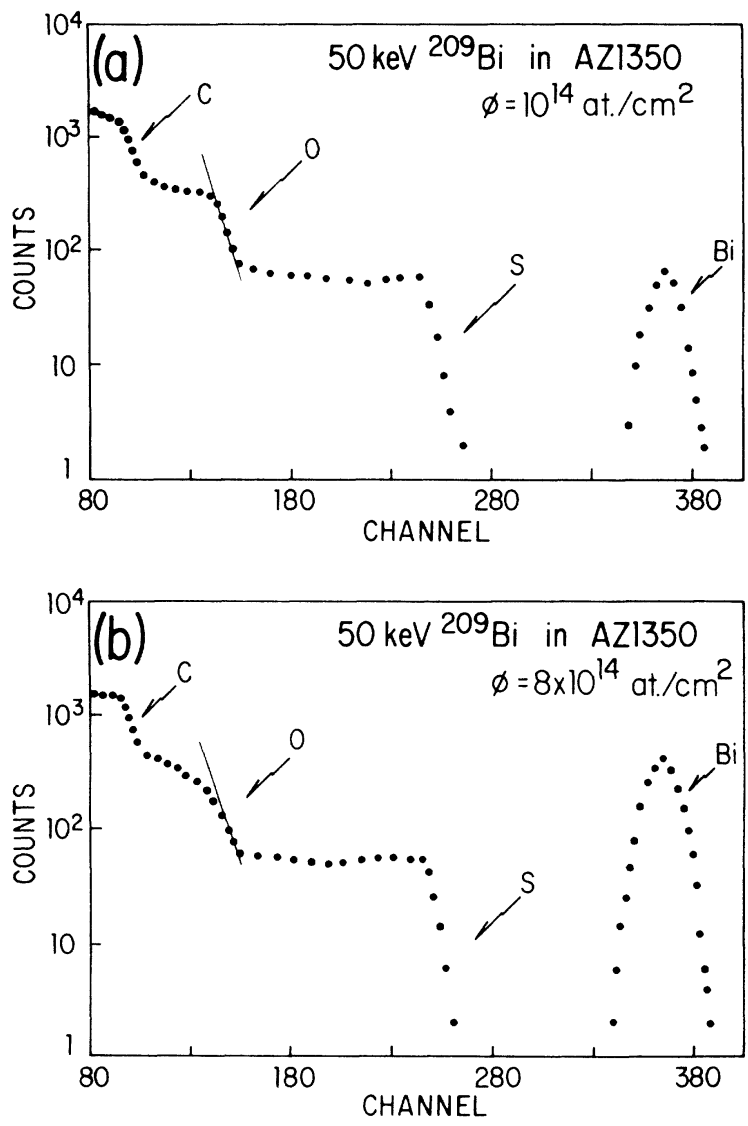

FIG. 1. (a) RBS spectrum of $50-\mathrm{keV}$ Bi implanted into AZ1350 photoresist with $10^{14}$ ions $/ \mathrm{cm}^{2}$. No significant loss of oxygen and/or carbon are observed. (b) RBS spectrum implanted at the same energy but with $\phi=8 \times 10^{14}$ at. $/ \mathrm{cm}^{2}$. There is a $\cong 35 \%$ oxygen loss in the region from the surface up to $700 \AA$. 
TABLE II. Experimental and TRIM calculated projected ranges $R_{p}$ and projected range stragglings $\Delta R_{p}$ for ${ }^{209} \mathrm{Bi}$ implanted into the $\mathrm{AZ} 1350$ photoresist film.

\begin{tabular}{ccccc}
\hline $\begin{array}{c}\text { Energy } \\
(\mathrm{keV})\end{array}$ & $\begin{array}{c}R_{p}(\mathrm{expt}) \\
(\AA)\end{array}$ & $\begin{array}{c}\Delta R_{p}(\mathrm{expt}) \\
(\AA)\end{array}$ & $\begin{array}{c}R_{p}(\mathrm{TRIM}) \\
(\AA)\end{array}$ & $\begin{array}{c}\Delta R_{p}(\mathrm{TRIM}) \\
(\AA)\end{array}$ \\
\hline 20 & 280 & 50 & 220 & 35 \\
30 & 350 & 70 & 280 & 40 \\
50 & 480 & 100 & 380 & 60 \\
100 & 730 & 120 & 580 & 80 \\
200 & 1110 & 200 & 930 & 130 \\
300 & 1550 & 300 & 1250 & 190 \\
\hline \hline
\end{tabular}

$\mathrm{Bi} / \mathrm{cm}^{2}$ we have detected a $35 \%$ oxygen loss from the surface up to $\cong 700 \AA$, as is displayed by Fig. 1(b). As shown by Table I the oxygen loss is proportional to the implantation dose. Nevertheless we obtain the important result that the range parameters are rather insensitive to the material loss as a consequence of the irradiation.

\section{B. Range measurements}

The $R_{p}$ and $\Delta R_{p}$ range parameters have been calculated using the Monte Carlo simulation TRIM code ${ }^{4}$ (1987 version). As mentioned before, it uses as inputs the universal potential proposed by Ziegler and Biersack ${ }^{1}$ and an improved electronic stopping power based on the work of Brandt and Kitagawa. ${ }^{3}$ Tables II and III show the $\mathrm{Au}$ and $\mathrm{Bi}$ experimental $R_{p}$ and $\Delta R_{p}$ values together with the TRIM predictions. The results are also displayed in Figs. 2 and 3, where the solid lines represent the TRIM theoretical predictions and the points correspond to the experimental results. Both figures clearly show that the experimental $R_{p}$ and $\Delta R_{p}$ values are always larger than the theoretical ones, with the difference being almost energy independent. For $\mathrm{Bi}$ the experimental $R_{p}$ values typically exceed the theoretical ones by $25 \%$, while for $\mathrm{Au}$ the difference is somewhat lower. Concerning the projected range stragglings $\Delta R_{p}$ the same kind of behavior is observed, with the experimental results exceeding the theoretical ones for as much as $75 \%$.

In our experimental range evaluation as well as for the theoretical TRIM code calculation, we have used for the density of the AZ1350 film, as given by the supplier, ${ }^{11}$ $\delta=1.3 \mathrm{~g} / \mathrm{cm}^{3}$. In addition to the evaluation of the stopping power and range calculations we have taken the composition of the AZ1350 photoresist as $\mathrm{C}_{6.2} \mathrm{H}_{6} \mathrm{O}_{1} \mathrm{~N}_{0.15} \mathrm{~S}_{0.06}{ }^{11}$ It is important to mention that the relative difference between the theoretical and experimental $R_{p}$ and $\Delta R_{p}$ values are independent of the assumed photoresist density.

\section{Thermal stability}

The RBS spectra of the nonimplanted samples show that up to $200^{\circ} \mathrm{C}$ there is no significant change in the chemical composition of the polymer film. However, for higher temperatures the situation changes drastically, as illustrated by Fig. 4, showing the RBS spectra corresponding to the nonannealed and annealed at 250 and $350^{\circ} \mathrm{C} \mathrm{AZ1350} \mathrm{samples.} \mathrm{At} 250^{\circ} \mathrm{C}$ there is a considerable loss of oxygen (around 20\%) for all the observed depth (from the surface up to $\cong 2000 \AA$ ). The corresponding C loss was of the order of $10 \%$. With increasing temperature a larger loss of material is observed and finally at $400{ }^{\circ} \mathrm{C}$ the photoresist is almost completely decomposed, being the $\mathrm{C}$ and $\mathrm{O}$ losses of the order of $28 \%$ and $50 \%$, respectively.

The $\mathrm{Au}$ implantations with $\phi=10^{14}$ and $3 \times 10^{14}$ at. $/ \mathrm{cm}^{2}$ and the Bi one with $\phi=10^{14}$ at. $/ \mathrm{cm}^{2}$ do not make any significant changes in the thermal behavior of the implanted AZ1350 samples. However, the situation changes when the AZ1350 films are $\mathrm{Bi}$ implanted with $\phi=3 \times 10^{14}$ at. $/ \mathrm{cm}^{2}$. Annealings up to $250^{\circ} \mathrm{C}$ do not produce any major change in the photoresist or in the $\mathrm{Bi}$ implanted profile. Annealing at $300^{\circ} \mathrm{C}$ results in small losses of oxygen (10\%) and carbon (5\%) (see Fig. 4) and a marked diffusion of the $\mathrm{Bi}$ implanted profile. At $350^{\circ} \mathrm{C}$ the $\mathrm{O}$ and $\mathrm{C}$ losses are $20 \%$ and $10 \%$, respectively, followed by a drastic diffusion of the Bi profile. Finally, at $400^{\circ} \mathrm{C}$ the photoresist partially decompose with further $\mathrm{O}$ and $\mathrm{C}$ losses of the order of $25 \%$ and $15 \%$, respectively.

The $\mathrm{C}$ and $\mathrm{O}$ losses as function of the temperature, for

TABLE III. Experimental and TRIM calculated projected ranges $R_{p}$ and projected range stragglings $\Delta R_{p}$ for ${ }^{197} \mathrm{Au}$ implanted into the $\mathrm{AZ} 1350$ photoresist film.

\begin{tabular}{|c|c|c|c|c|}
\hline $\begin{array}{c}\text { Energy } \\
(\mathrm{keV})\end{array}$ & $\begin{array}{c}R_{p}(\text { expt }) \\
\quad(\AA ̊) \\
\end{array}$ & $\begin{array}{c}\Delta R_{p} \text { (expt) } \\
\quad(\AA)\end{array}$ & $\begin{array}{cl}R_{p} & (\mathrm{TRIM}) \\
& (\AA \stackrel{\AA}{ }) \\
\end{array}$ & $\Delta R_{p}\left(\begin{array}{l}\text { (TRIM }) \\
(\AA)\end{array}\right.$ \\
\hline 20 & 290 & 50 & 225 & 35 \\
\hline 30 & 330 & 70 & 285 & 40 \\
\hline 50 & 450 & 80 & 390 & 60 \\
\hline 80 & 620 & 100 & 522 & 75 \\
\hline 100 & 720 & 120 & 605 & 90 \\
\hline 200 & 1180 & 230 & 1000 & 140 \\
\hline
\end{tabular}




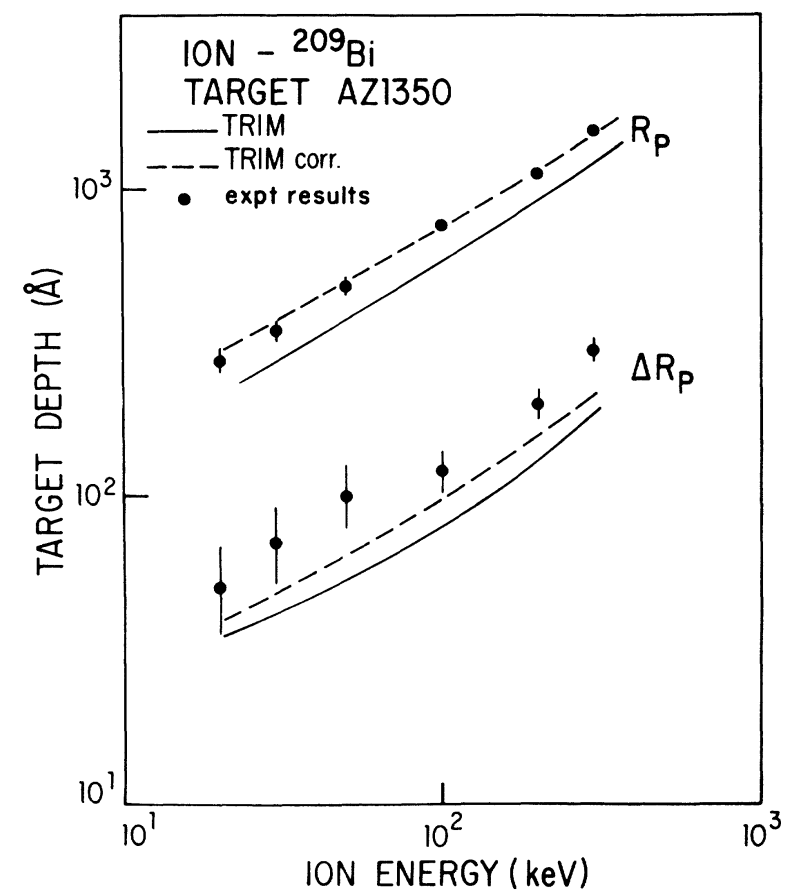

FIG. 2. Comparison of the ${ }^{209} \mathrm{Bi}$ experimental and calculated projected range $R_{p}$ and range straggling $\Delta R_{p}$ as a function of the energy. Solid points represent the experimental results. The solid lines correspond to the TRIM predictions. Dashed lines represent the modified TRIM calculations-see text.

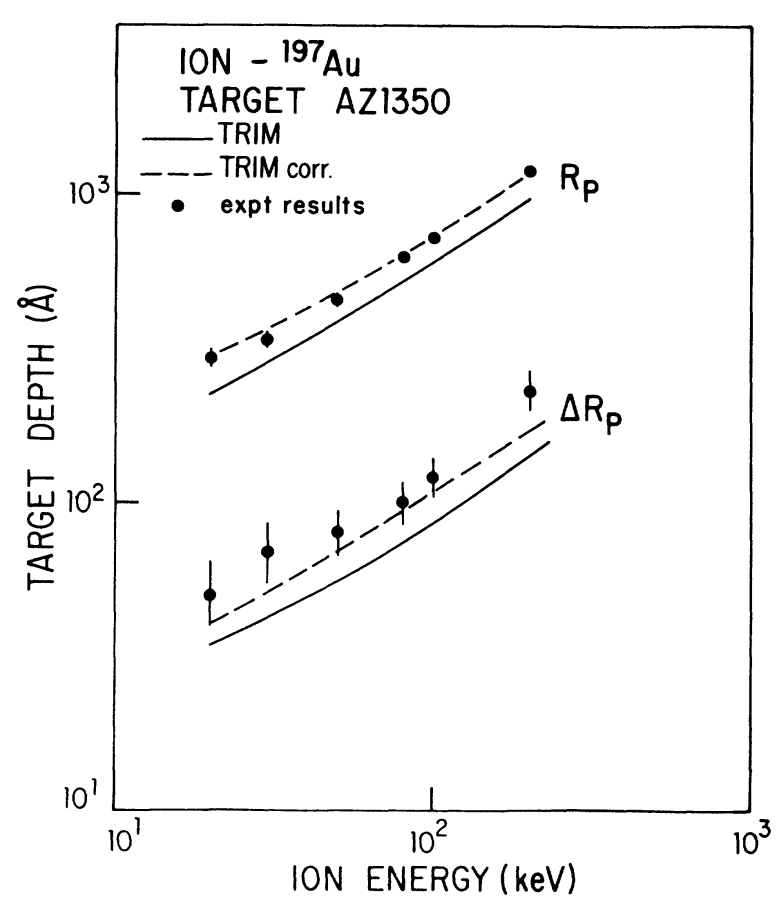

FIG. 3. Comparison of the ${ }^{197} \mathrm{Au}$ experimental and calculated projected range $R_{p}$ and range straggling $\Delta R_{p}$ as a function of the energy. Solid points represent the experimental results. The solid lines correspond to the TRIM predictions. Dashed lines represent the modified TRIM calculations-see text.

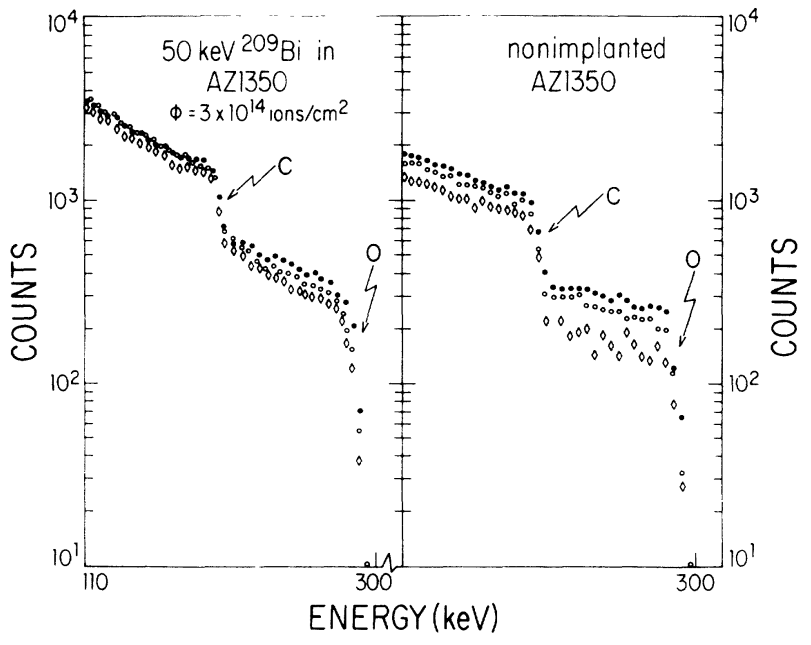

FIG. 4. The right-hand side shows the RBS spectra corresponding to unimplanted AZ1350 polymer film, as received (solid points), after $250^{\circ} \mathrm{C}$ annealing (open points), and after $350^{\circ} \mathrm{C}$ annealing (open diamond). The left-hand side shows the RBS spectra corresponding to a shallow $\phi=3 \times 10^{14} \mathrm{Bi} / \mathrm{cm}^{2} \mathrm{im}$ plantation into the polymer film, as implanted (solid points), after $300^{\circ} \mathrm{C}$ annealing (open points), and after $350^{\circ} \mathrm{C}$ annealing (open diamond).

the nonimplanted and the $\mathrm{Bi}$ and $\mathrm{Au}$ implanted $\mathrm{AZ} 1350$ samples, are summarized in Table IV.

\section{Diffusional behavior}

In addition to the thermal stability of the polymer, we have also studied the diffusional behavior of both $\mathrm{Au}$ and Bi implanted into the AZ1350 photoresist.

The analysis of the thermal diffusion experiments in organic polymer implanted systems cannot be performed in a straightforward way by analytical techniques. This is because here the implantation leads to irreversible damage that does not anneal out as in metals or semiconductors. To overcome this situation we have used a numerical approach of the so-called "finite difference method" 12 in order to analyze our data. In this approach the whole depth profile of a given distribution is divided into single channels. The diffusion of each channel is treated subsequently and the product of all diffused contributions of each channel is summed up in order to construct a new profile. Because of the channel by channel treatment any input profile can be "diffused" independently of its shape by using a depth-dependent diffusion parameter $D(x)$. For convenience we separate $D(x)$ into a depthindependent value $D^{*}$ and a normalized dimensionless depth-dependent function $f(x)=D(x) / D^{*}$.

Figure 5 shows the result obtained after the application of the aforementioned procedure. The solid points represent the $\phi=10^{14} \mathrm{Bi} / \mathrm{cm}^{2}$ depth profile of the $200^{\circ} \mathrm{C}$ annealed sample. The open points show the ion distribution after annealing at $300^{\circ} \mathrm{C}$. The inset in Fig. 5 displays the depth-dependent $f(x)$ function used as input by the computer program to reproduce the final ion distribution profile. The depth dependence of $f(x)$ was chosen fol- 
TABLE IV. Carbon $(\mathrm{C})$ and oxygen $(\mathrm{O})$ relative losses (in \%) as a function of the annealing temperature. Results for both implanted ions and fluences.

\begin{tabular}{|c|c|c|c|c|c|c|c|c|c|c|}
\hline \multirow{4}{*}{$\begin{array}{c}\text { Temperature } \\
\left({ }^{\circ} \mathrm{C}\right)\end{array}$} & \multicolumn{2}{|c|}{ Nonimplanted } & \multicolumn{8}{|c|}{ Implanted } \\
\hline & \multirow[b]{3}{*}[\mathrm{C}]{$(\%)$} & \multirow[b]{3}{*}[\mathrm{O}]{$(\%)$} & \multicolumn{4}{|c|}{$\left(\phi=10^{14}\right.$ at.$\left./ \mathrm{cm}^{2}\right)$} & \multicolumn{4}{|c|}{$\left(\phi=3 \times 10^{14}\right.$ at. $\left./ \mathrm{cm}^{2}\right)$} \\
\hline & & & \multicolumn{2}{|c|}{$\mathrm{Au}$} & \multicolumn{2}{|c|}{$\mathrm{Bi}$} & \multicolumn{2}{|c|}{$\mathrm{Au}$} & \multicolumn{2}{|c|}{$\mathrm{Bi}$} \\
\hline & & & {$[\mathrm{C}](\%)$} & {$[\mathrm{O}](\%)$} & {$[\mathrm{C}](\%)$} & {$[\mathrm{O}](\%)$} & {$[\mathrm{C}](\%)$} & {$[\mathrm{O}](\%)$} & {$[\mathrm{C}](\%)$} & {$[\mathrm{O}](\%)$} \\
\hline 250 & 10 & 20 & 10 & 25 & & & 4 & 16 & & \\
\hline 300 & 15 & 25 & 20 & 40 & 20 & 35 & 10 & 28 & & 10 \\
\hline 350 & 20 & 40 & 20 & 50 & 30 & 50 & 20 & 30 & 10 & 20 \\
\hline 400 & 28 & 50 & 30 & 60 & 30 & 53 & 25 & 40 & 15 & 25 \\
\hline
\end{tabular}

lowing the Monte Carlo TRIM code calculations ${ }^{4}$ which predict for the present case a damaged region going from the surface to around $400 \AA$ depth.

Starting from the initial range profile and applying the $f(x)$ parameter, the program after several iterations provides the profile shown in Fig. 5 as a full line. The agreement between the calculated and experimental results is quite satisfactory, in particular for the near surface and bulk regions. The extracted diffusion coefficients for $\mathrm{Bi}$ in the damaged and bulk regions are, respectively, $D_{d}=1.7 \times 10^{-15} \mathrm{~cm}^{2} / \mathrm{sec}$ and $D_{b}=1.7 \times 10^{-14} \mathrm{~cm}^{2} / \mathrm{sec}$.

This calculation has been repeated for $\mathrm{Bi}$ and $\mathrm{Au}$ for all the studied temperatures and implanted fluences. The annealing temperatures and corresponding $D_{d}$ and $D_{b}$ diffusion coefficients are displayed in Table V. It should be noted that the bulk diffusion coefficients are independent of the Bi or Au implantation dose.

\section{DISCUSSION}

\section{A. Range data}

It is well known that energetic ions damage and modify organic materials in a chemically irreversible fashion. However, the present work has shown that the range parameters are rather insensitive to local stoichiometric modifications produced by the implantation process.

The experimental $\mathrm{Au}$ and $\mathrm{Bi}$ range parameters deduced in the present work are always larger than the $\mathrm{ZBL}$ predictions. The differences are beyond the experimental errors and cannot be attributed to the lack of precise knowledge of the polymer density. As was pointed out before, the relative difference between the experimental and theoretical values is independent of the assumed density.

A source for the observed disagreement could be related to the composition of the polymer, which was taken as $\mathrm{C}_{6.2} \mathrm{H}_{6} \mathrm{O}_{1} \mathrm{~N}_{0.15} \mathrm{~S}_{0.06}$. If this is not the real stoichiometry, changes in both the theoretical and experimental range parameters should be expected. However, reasonable modifications of the nominal composition (around 10\% for each component) gives a variation of only $2 \%$ to $5 \%$ in the theoretical-experimental discrepancies.

A second source of disagreement could be associated with the validity of the Bragg's rule for organic and related materials. A recent survey ${ }^{13}$ has shown a general failure of the simple additivity rule for light ions like $\mathbf{H}$, $\mathrm{He}$, and $\mathrm{Li}$. Differences of the order 5-10\% and some times up to $25 \%$ have been observed at the stoppingpower peak energy. Concerning heavy ions, the information is scarce and nothing definitive can be said. In our

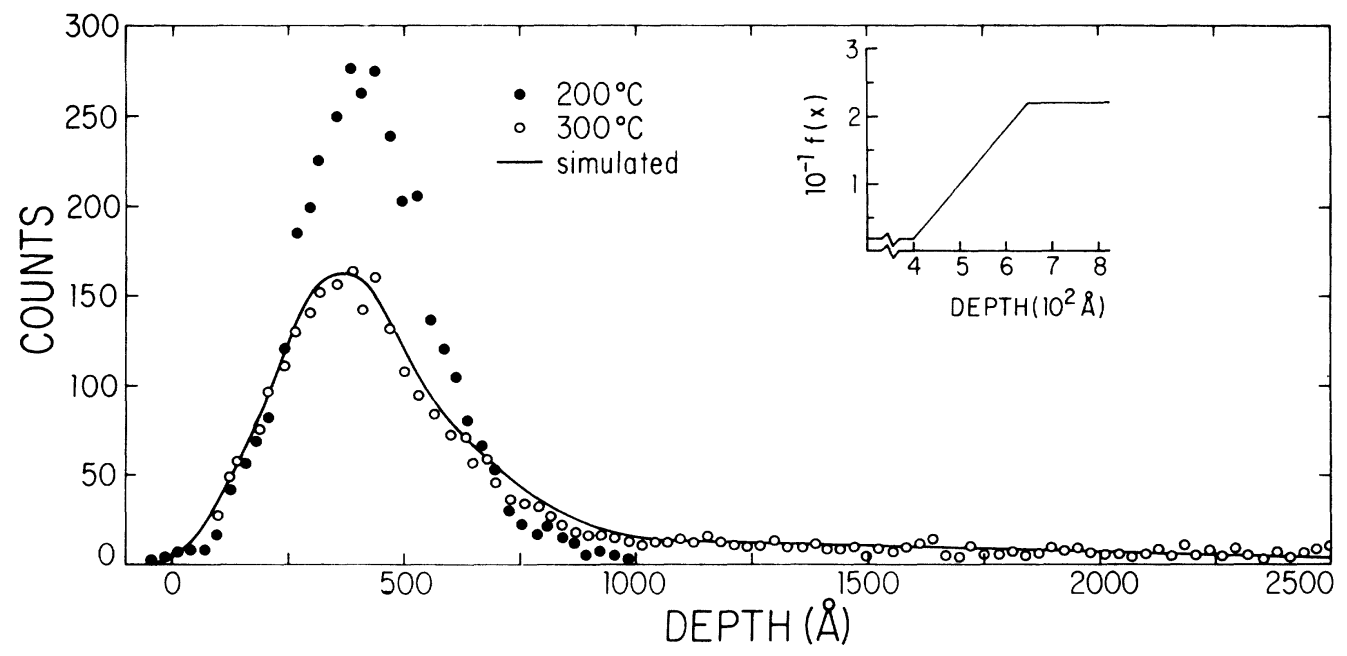

FIG. 5. Solid points represent the Bi depth profile after $200^{\circ} \mathrm{C}$ annealing. The open points show the ion distribution after annealing at $300^{\circ} \mathrm{C}$. The inset displays the depth-dependent diffusion parameter used in the calculation. The smooth curve represents the calculated depth profile. 
TABLE V. $D_{d}$ and $D_{b}$ diffusion coefficients (see text) as a function of the temperature for Au and Bi at the two implanted fluences. The bulk diffusion coefficients $D_{b}$ are independent of the implanted fluence.

\begin{tabular}{|c|c|c|c|c|c|c|}
\hline \multirow{2}{*}{$\begin{array}{c}\text { Temperature } \\
(\mathbf{K})\end{array}$} & \multicolumn{2}{|c|}{$\phi=10^{14}$ at. $/ \mathrm{cm}^{2}$} & \multicolumn{2}{|c|}{$\phi=3 \times 10^{14}$ at. $/ \mathrm{cm}^{2}$} & \multicolumn{2}{|c|}{$\begin{array}{l}\text { Bulk diffusion } \\
\text { coefficients }\end{array}$} \\
\hline & \multicolumn{2}{|c|}{$D_{d}\left(\mathrm{~cm}^{2} / \mathrm{sec}\right)$} & \multicolumn{2}{|c|}{$D_{d}\left(\mathrm{~cm}^{2} / \mathrm{sec}\right)$} & \multicolumn{2}{|c|}{$D_{b}\left(\mathrm{~cm}^{2} \mathrm{sec}\right)$} \\
\hline 473 & $6.5 \times 10^{-17}$ & $5.0 \times 10^{-16}$ & $2.3 \times 10^{-16}$ & $8.0 \times 10^{-17}$ & $6.5 \times 10^{-16}$ & $7.0 \times 10^{-15}$ \\
\hline 573 & $6.2 \times 10^{-16}$ & $1.7 \times 10^{-15}$ & $1.3 \times 10^{-16}$ & $4.0 \times 10^{-16}$ & $7.0 \times 10^{-15}$ & $1.7 \times 10^{-14}$ \\
\hline 623 & $4.0 \times 10^{-16}$ & $2.5 \times 10^{-15}$ & $2.6 \times 10^{-17}$ & $7.5 \times 10^{-16}$ & $4.0 \times 10^{-14}$ & $2.0 \times 10^{-14}$ \\
\hline 673 & $5.0 \times 10^{-16}$ & $4.0 \times 10^{-15}$ & $1.0 \times 10^{-16}$ & $1.6 \times 10^{-15}$ & $2.0 \times 10^{-15}$ & $3.2 \times 10^{-14}$ \\
\hline
\end{tabular}

experiment we have applied the Bragg's rule twice: first, when we calculated the stopping power of the analyzing beam in the AZ1350 film; second, when the TRIM was used in order to simulate the implanted profiles and extract the corresponding range parameters.

As an alternative to the Bragg's rule, Ziegler and Manoyen ${ }^{14}$ have recently developed a simple method for calculating the stopping of ions in compounds based on details of the molecular bonding of the material (C.A.B. model). We have applied the C.A.B. calculations to the present case and found the following differences with those from Bragg's rule: $5 \%$ for the $\alpha$ stopping and less than $2 \%$ for the $\mathrm{Au}$ and $\mathrm{Bi}$ projected ranges. As can be observed, these differences might account only in part for the theoretical-experimental discrepancies.

There is a third possibility that we want to discuss in detail. Recently Grande et al. ${ }^{15}$ have reported profile measurements for heavy ions implanted into $\mathrm{C}$ films. The extracted range parameters have always shown to be significantly larger (typically 30\%) than the ZBL predictions, the difference being independent of the implantation energy. Grande et al. ${ }^{16}$ have tried different approaches, e.g., the type of potential was changed and the electronic stopping power was modified but no one has improved the agreement between the theoretical and experimental data. However, good agreement was achieved when a correlation between the nuclear and electronic stopping powers was included in the ZBL calculations.

The present case is very similar to the $C$ one. We have implanted heavy ions into a substrate composed by light elements (basically $\mathrm{C}, \mathrm{O}$, and $\mathrm{H}$ ) and we observed once more that the experimental values are significantly larger than the $\mathrm{ZBL}$ calculation. These facts seem to confirm the prediction of Grande et al. ${ }^{16}$ stating that this feature appears whenever heavy ions are implanted into light substrates. It will now be interesting to see if the theoretical procedure developed in Ref. 16 is suitable for the present case. In what follows we are going to summarize this procedure and apply it to the present case.

In the $\mathrm{ZBL}$ original calculation a common simplification is done by taking the elastic $\left(S_{n}\right)$ and in- elastic $\left(S_{e}\right)$ transfer energy process as uncorrelated. Then

$$
S_{t}=S_{n}^{\text {uncorr }}+S_{e}
$$

consequently the energy transferred to a target atom in an elastic binary collision with impact parameter $b$ is given by

$$
T_{b}^{\mathrm{uncorr}}=T_{m} \sin ^{2} \frac{\theta}{2},
$$

where $T_{m}$ is the maximum transferred energy and $\theta$ is the c.m. scattering angle. When inelastic effects are taken into account, (1) is transformed into

$$
T(b)^{\mathrm{corr}}=T_{m}\left[f \sin ^{2} \theta / 2+0.25(1-f)^{2}\right],
$$

with

$$
f=\sqrt{1-Q(b) / E_{\mathrm{c} . \mathrm{m} .}},
$$

being $Q(b)$ the inelastic energy loss and $E_{\text {c.m. }}$ the c.m. energy.

To first order in $Q / E_{\text {c.m. }}$, the expression (2) is reduced to

$$
T(b)^{\mathrm{corr}}=\gamma E \sin ^{2} \frac{\theta}{2}-2 \beta Q(b) \sin ^{2} \frac{\theta}{2},
$$

and the total energy loss of the ion becomes

$$
\Delta E(b)=\gamma E \sin ^{2} \frac{\theta}{2}+Q(b)-2 \beta Q(b) \sin ^{2} \frac{\theta}{2},
$$

with

$$
\gamma=4 \frac{m_{1} m_{2}}{\left(m_{1}+m_{2}\right)^{2}} \quad \text { and } \beta=\frac{m_{1}}{m_{1}+m_{2}} .
$$

In the usual stopping theories the last term of (3) is neglected and in addition it is assumed that $\theta$ is independent of $Q(b)$. This last hypothesis is not strictly correct, in particular when close collisions occur.

The dependence of $\theta$ with $Q(b)$ is calculated by using the electron promotion model described in Refs. 17 and 18. As a result it can be shown that

$$
\begin{aligned}
\theta(\epsilon, b)= & {\left[\frac{\pi}{2}-\int_{r_{0}}^{\infty} \frac{d r b}{r^{2}\left[1-V_{\mathrm{ZBL}} / E_{\mathrm{c} . \mathrm{m} .}-(b / r)^{2}\right]^{1 / 2}}\right]_{\text {incoming path }} } \\
& \left.+\int \frac{\pi}{2}-\int_{r_{0}}^{\infty} \frac{d r b}{r^{2}\left[1-V_{\mathrm{exc}} / E_{\mathrm{c} . \mathrm{m} .}-(b / r)^{2}\right]^{1 / 2}}\right]_{\text {outgoing path }},
\end{aligned}
$$


with $\epsilon, b$, and $r_{0}$ defined as usual. ${ }^{4}$ The incoming path in the c.m. system is not modified and can be calculated using the ZBL potential. ${ }^{2}$ After the collision, the outgoing path corresponds to an excited $V_{\text {exc }}$ state potential. As discussed in Ref. 16 by taking

$$
V_{\mathrm{exc}}(r)=\lambda V_{\mathrm{ZBL}}(r)+Q(b)
$$

with

$$
\lambda=1=\frac{Q(b)}{V_{\mathrm{ZBL}}\left(r_{0}\right)}
$$

(4) is transformed into

$$
\theta(\epsilon, b)=\left[\frac{\theta^{\mathrm{ZBL}}(\epsilon, b)+\theta^{\mathrm{ZBL}}\left(\epsilon^{\prime}, b^{\prime}\right)}{2}\right\rfloor
$$

with

$$
\epsilon^{\prime}=\epsilon \frac{\left(1-Q / E_{\text {c.m. }}\right)}{\lambda}, b^{\prime}=\frac{b}{\sqrt{(1-Q / \text { c.m. })}},
$$

and

$$
\theta^{\mathrm{ZBL}}(\epsilon, b)=\pi-2 \int_{r_{0}}^{\infty} \frac{d r b}{r^{2}\left[1-V_{\mathrm{ZBL}} / E_{\text {c.m. }}-(b / r)^{2}\right]^{1 / 2}} .
$$

This is an essential point in the treatment since it allows the use of the ZBL magic formula ${ }^{2}$ for (5) not only for the incoming but also for the outgoing path.

In the next step we calculated the total stopping power for $\mathrm{Bi}$ and $\mathrm{Au}$ implanted into AZ1350 film by taking into account the inelastic effects on the nuclear stopping power $\mathrm{Sn}$. In this calculation we use the following expression for $Q(b)$ :

$$
Q(b)=Q_{\text {Inner }}(b)+Q_{v}(b),
$$

where $Q_{\text {Inner }}$ is the energy loss due to inner electron and $Q_{v}$ is due to the valence electrons. For $Q_{v}(b)$ the expression proposed by Oen and Robinson ${ }^{19}$ can be used,

$$
Q(b)_{v}=S_{e}^{v}(2 \pi)^{-1} \alpha^{2} \exp \left(-\alpha \frac{r_{0}}{a_{u}}\right),
$$

where $S_{e}^{v}$ is the ZBL electronic cross section, $\alpha$ is an empirical parameter taken as 0.5 , and $a_{u}$ is the universal screening length. ${ }^{2}$

Concerning $Q_{\text {Inner }}(b)$, it can be assumed that the candidates for electron promotion are the carbon and oxygen $K$ shell electrons and for the heavy impinging atoms, the $N$ shell electrons. The corresponding excitation energies and critical distances deduced from Ref. 20 are displayed in Table VI. For $\mathrm{Bi}$ and $\mathrm{Au}$ light system substrates this is the best that can be done since it is very hard for this systems to perform molecular orbital calculations. With the preceding excitation energies $Q(b)$ and critical distances $r_{0}$ it is possible to calculate the correlated nuclear stopping cross section

$$
S_{n}^{\mathrm{corr}}=\int 2 \pi b T(b)^{\mathrm{corr}} d b
$$

through expressions (5) and (2). Then the total correlated stopping power becomes
TABLE VI. Inelastic energy loss $Q$ and critical distance for each ion-atom combination.

\begin{tabular}{ccc}
\hline \hline ion-atom & $\begin{array}{c}Q_{\text {Inner }} \\
(\mathrm{eV})\end{array}$ & $r_{c} / a_{u}{ }^{\mathrm{a}}$ \\
\hline Au-C & 660 & 3.6 \\
Au-O & 800 & 3.0 \\
Bi-C & 760 & 3.4 \\
Bi-O & 900 & 2.8 \\
\hline \hline
\end{tabular}

${ }^{\mathrm{a}} a_{u}$ is the ZBL screening length.

$$
S_{T}^{\text {corr }}=S_{n}^{\text {corr }}+S_{e} \text { with } S_{e}=S_{e_{\text {Inner }}}+S_{e}^{v} .
$$

The results of the calculations are displayed as dashed lines in Figs. 2 and 3, showing that a good agreement is achieved when the original ZBL calculations are modified by the inclusion of inelastic effects in the nuclear stopping power.

\section{B. Thermal stability}

The results of the present experiment show (see Table IV) that the $\mathrm{Au}$ implantations do not produce any significant improvement in the thermal stability of the AZ1350 film. The amount of carbon and oxygen lost by the $10^{14} \mathrm{Au} / \mathrm{cm}^{2}$ implanted sample is similar if not a little bit larger than that of the nonimplanted one. These results are observed in Table IV and in Fig. 6, where the $O$ and $\mathrm{C}$ losses are displayed for each implanted $\mathrm{Au}$ or $\mathrm{Bi}$ fluence as a function of the annealing temperature. For $\phi=3 \times 10^{14} \mathrm{Au} / \mathrm{cm}^{2}$ the $\mathrm{O}$ and $\mathrm{C}$ losses decrease but still are comparable with those corresponding to the nonimplanted sample. The Bi implanted sample with $\phi=10^{14}$ at. $/ \mathrm{cm}^{2}$ seems to be stable up to $250^{\circ} \mathrm{C}$, but for higher temperatures loses even more material $(\mathrm{C}$ and $\mathrm{O})$ than the nonimplanted one. However, for $\phi=3 \times 10^{14}$ there is a drastic reduction in the material loss as shown by Fig. 7 and Table IV and a significant decomposition of the photoresist starts at only around $350^{\circ} \mathrm{C}$.

Guimarães et al. ${ }^{8}$ have suggested that the damage created by the implantation could be responsible for the raising of the temperature at which the photoresist starts to decompose. The results of the present experiment clearly show that the damage is not the only mechanism responsible for the preceding effect. $\mathrm{Bi}$ and $\mathrm{Au}$ produce the same damage. However, at $\phi=3 \times 10^{14}$ at. $/ \mathrm{cm}^{2}$ only $B i$ is really efficient in preventing the dissolution of the photoresist. This means that in addition to radiation damage, chemical effects very likely due to the bonding of $\mathrm{Bi}$ with molecules of the photoresist, play an important role in its thermal behavior.

Our results also show that there is a threshold implantation fluence $\phi_{\mathrm{th}}$ needed to inhibit the decomposition of the photoresist. Implantation fluences lower than $\phi_{\text {th }}$ seem to accelerate the decomposition, as shown by Fig. 6 . For $\mathrm{Au}$ this statement is valid for the $200-400^{\circ} \mathrm{C}$ thermal range, while for $\mathrm{Bi}$ the effect starts at $300^{\circ} \mathrm{C}$. The $\phi_{\text {th }}$ depends on the characteristic of the photoresist. In the case of the AZ111 this threshold fluence $\phi_{\text {th }}$ was reported to be $\phi=10^{14} \mathrm{Bi} / \mathrm{cm}^{2}$, while in the present case it is higher, 

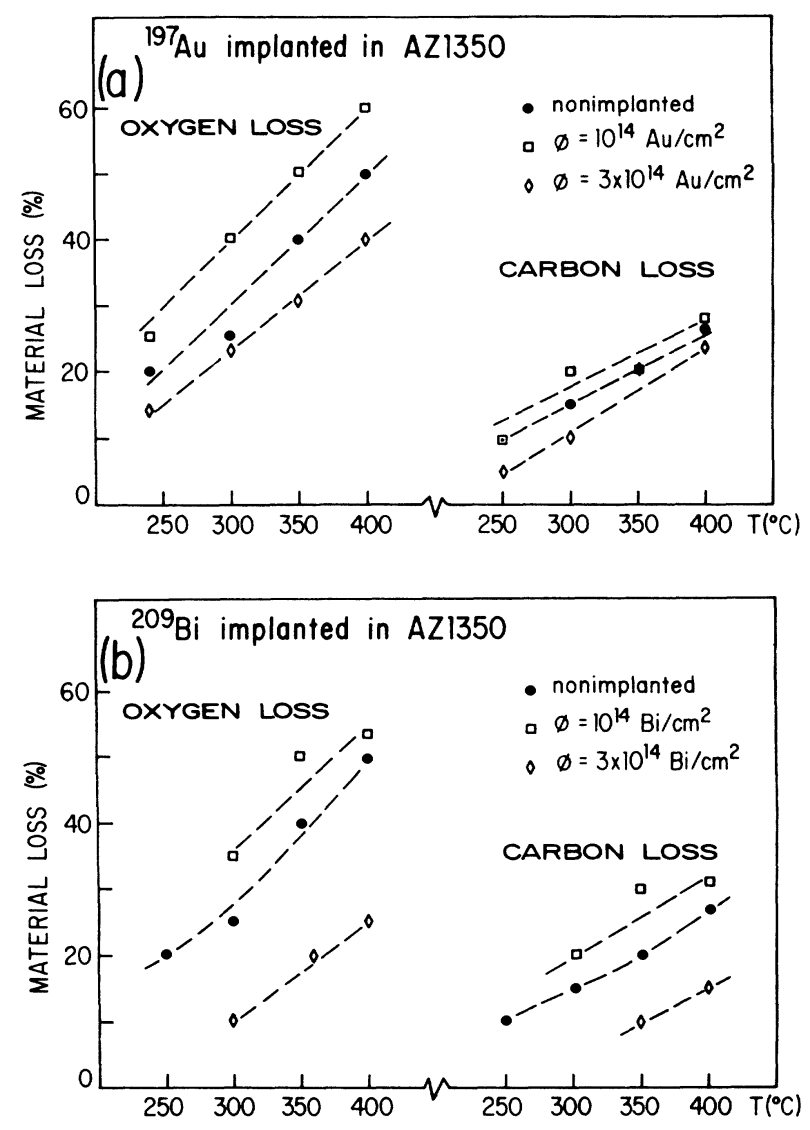

FIG. 6. (a) Oxygen and carbon losses as a function of the temperature for a nonimplanted AZ1350 samples, Au implanted with $\phi=10^{14}$ and $3 \times 10^{14}$ at. $/ \mathrm{cm}^{2}$. It should be observed that the carbon loss at $250^{\circ} \mathrm{C}$ for the unimplanted and $\phi=10^{14}$ $\mathrm{Au} / \mathrm{cm}^{2}$ samples are similar. The same appears at $350^{\circ} \mathrm{C}$ for the nonimplanted, $\phi=10^{14}$ and $3 \times 10^{14} \mathrm{Au} / \mathrm{cm}^{2}$ samples. (b) Same as (a) but the AZ1350 samples are implanted with $\mathrm{Bi}, \phi=10^{14}$ and $3 \times 10^{14}$ at. $/ \mathrm{cm}^{2}$.

around $\phi=3 \times 10^{14} \mathrm{Bi} / \mathrm{cm}^{2}$. Both photoresists have the same stoichiometric composition; however, their structure, density, and viscosity are different. This could be a reason for the differences in the observed $\phi_{\text {th }}$ needed to stabilize the polymer at higher temperatures.

\section{Diffusional behavior}

The results displayed in Table $\mathrm{V}$ indicate that $\mathrm{Bi}$ diffuses regularly in the undamaged as well as in the damaged region during the annealing of the AZ1350 photoresist.

In Fig. 7 the $\mathrm{Bi}$ diffusion coefficients $D_{d}$ and $D_{b}$ are displayed as a function of $T^{-1}$. For both fluences the experimental $D_{d}$ points follow straight lines, indicating an Arrhenius-type behavior. The extracted activation energies are $E_{b}=310$ and $420 \mathrm{meV}$ for the $10^{14}$ and $3 \times 10^{14}$ fluences, respectively. The same kind of behavior is followed by the bulk diffusion coefficients $D_{b}$-see Fig. 7 . In this case, as expected, the extracted coefficients are in-

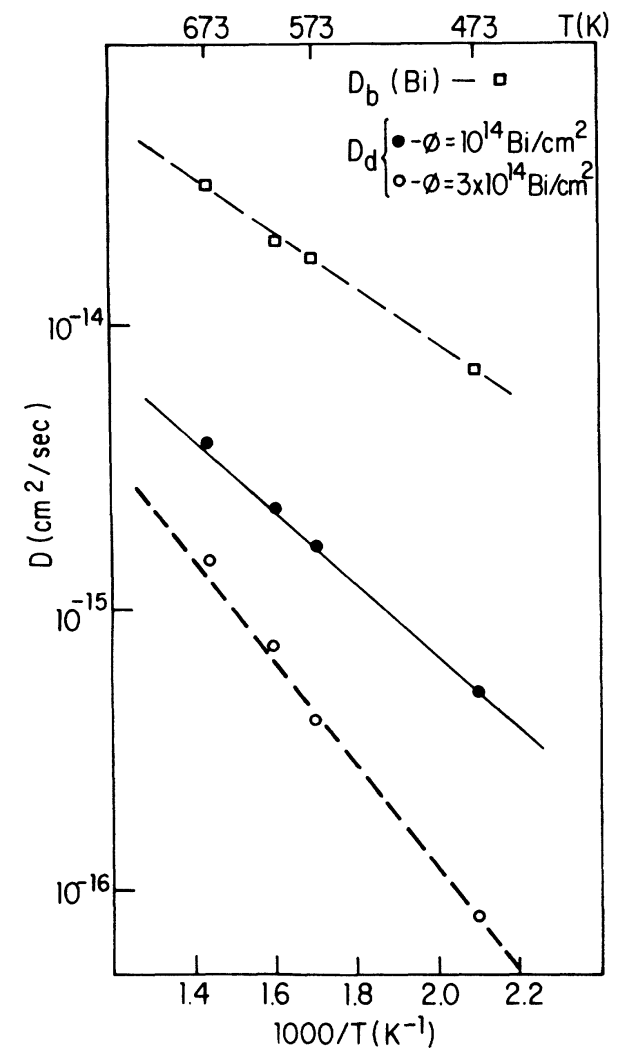

FIG. 7. Bi diffusion $D_{d}$ coefficients in the AZ1350 photoresist for $\phi=10^{14}$ and $3 \times 10^{14} \mathrm{Bi} / \mathrm{cm}^{2}$ as a function of $1 / T$. Also are shown the $\mathrm{Bi} D_{b}$ diffusion coefficients. The lines are only drawn to guide the eye. In all the cases the Bi diffuses regularly.

dependent of the implantation fluence, being the corresponding activation energy value $E_{b}=220 \mathrm{meV}$. The differences in the activation energy values are related to the damage produced by the implantation process. The higher the fluence, the larger the damage, and therefore one expects that the activation energy of the $\mathrm{Bi}$ ions would be higher in agreement with what is observed in the experiment. Moreover, the lowest $E_{b}=220 \mathrm{meV}$ value corresponds to the diffusion in the undamaged region.

On the other side, Table $\mathrm{V}$ shows that the Au data do not follow a regular diffusional process for any of the implanted fluences. The differences in the thermal behavior of $\mathrm{Au}$ and $\mathrm{Bi}$ can be, in principle, attributed to the different $\mathrm{Au}$ and $\mathrm{Bi}$ chemical bonds with the elements of the photoresist.

\section{CONCLUSIONS}

Our range profile results have shown that the ZBL predictions are significantly lower than the experimental values. Between the various hypotheses raised in this paper, we cannot disregard the one related to the validity of the Bragg's rule. As mentioned earlier a recent survey has shown that for light ions implanted into organic materials, the average uncertainty in the Bragg's rule is of 
the order of $5-10 \%$. As an alternative to the Bragg's rule we have followed the procedure suggested by Ziegler and Manoyan ${ }^{14}$ but we did not get a significant improvement in the theoretical predictions. Therefore the uncertainty introduced by the application of the Bragg's rule still should be left as an open question.

The hypothesis related to the inclusion of inelastic effects in the theoretical nuclear stopping power seems to be more reliable. In fact, this hypothesis fits with an emerging picture that shows that whenever heavy ions are implanted into light substrates the experimental range parameters exceed significantly the ones proposed by the ZBL theory. Moreover, the inclusion of the correlation between the inelastic and elastic transfer energy processes in the ZBL calculations bring as a consequence good agreement with the experimental data. Nevertheless, further work is needed in order to confirm the aforemen- tioned hypothesis.

Concerning the thermal behavior of the implanted polymer it was shown that not only radiation damage but also chemical effects are important in raising the decomposition temperature of the photoresist. Present and previous experiments have shown that a threshold fluence of $\mathrm{Bi}$ is necessary in order to achieve the stabilization effect. This threshold fluence seems to be a function of the polymer used in the experiment. Finally, our work has shown that the diffusional behavior of the implanted ions is strongly dependent on the characteristic of the ion and on the implantation process itself. While $\mathrm{Bi}$ diffuses regularly, independent of the implantation dose, Au does not follow an Arrhenius behavior. In addition, it was shown that the implantation process modifies, via the nonrecovered damage, the characteristics of the $\mathrm{Bi}$ diffusional process.
1J. P. Biersack and J. F. Ziegler, in Ion Implantation Techniques, edited by $\mathrm{H}$. Ryssel and $\mathrm{H}$. Glawischnig (Springer, Berlin, 1982).

${ }^{2}$ J. F. Ziegler, J. P. Biersack, and U. Littmark, in The Stopping and Ranges of Ions in Solids, edited by J. F. Ziegler (Pergamon, New York, 1985).

${ }^{3}$ W. Brandt and M. Kitagawa, Phys. Rev. B 25, 5631 (1982).

${ }^{4} J$. P. Biersack and L. G. Haggmark, Nucl. Instrum. Methods 178, 257 (1980).

${ }^{5}$ R. B. Guimarães, L. Amaral, M. Behar, P. F. P. Fichtner, F. C. Zawislak, and D. Fink, J. Appl. Phys. 63, 1083 (1988).

${ }^{6}$ R. B. Guimarães, L. Amaral, M. Behar, D. Fink, and F. C. Zawislak, J. Mater. Res. 3, 1422 (1988).

${ }^{7}$ Y. Okayama, T. Hashimoto, and T. Yoguch, J. Electrochem. Soc. 125, 1293 (1988).

${ }^{8}$ R. B. Guimarães, L. Amaral, R. P. Livi, J. P. de Souza, M. Behar, and F. C. Zawislak, Nucl. Instrum. Methods B32, 419 (1988).

${ }^{9}$ W. K. Chu, J. W. Mayer, and M. A. Nicolet, Backscattering Spectrometry (Academic, New York, 1978).
${ }^{10}$ D. C. Santry and R. D. Werner, Nucl. Instrum. Methods 178, 523 (1980).

${ }^{11}$ Shypley, Co., Zürich, Switzerland.

${ }^{12}$ G. D. Smith, Numerical Solution of Partial Differential Equations: Finite Difference Method, 2nd ed. (Clarendon, Oxford, 1978).

${ }^{13}$ D. I. Thwaites, Nucl. Instrum. Methods B27, 293 (1987), and reference therein.

${ }^{14}$ J. F. Ziegler and J. M. Manoyan, Nucl. Instrum. Methods B35, 215 (1988).

${ }^{15}$ P. L. Grande, P. F. P. Fichtner, M. Behar, and F. C. Zawislak, Nucl. Instrum. Methods B33, 122 (1988).

${ }^{16}$ P. L. Grande, M. Behar, G. Schiwietz, and J. P. Biersack, private communication.

${ }^{17}$ U. Fano and W. Lichten, Phys. Rev. Lett. 14, 627 (1966).

${ }^{18}$ U. Wille and R. Hippler, Phys. Rep. 132, 129 (1986), and reference therein.

${ }^{19} \mathrm{O}$. Oen and M. Robinson, Nucl. Instrum. Methods 132, 647 (1976).

20J. P. Desclaux, At. Data Nucl. Data Tables 12, 311 (1973). 